\title{
Unusual left ventricular aneurysm in a patient with anomalous origin of left coronary artery from pulmonary artery
}

\author{
P. Kafkas ${ }^{1}$ and G. A. H. Miller \\ From the Cardiac Department, Brompton Hospital, London S.W.3
}

A case is described of a I3-year-old girl with anomalous origin of the left coronary artery from the pulmonary artery, in whom an unusual left ventricular aneurysm was demonstrated angiographically.

Anomalous origin of the left coronary artery from the pulmonary artery is a rare condition accounting for some 0.5 per cent of cases of congenital heart disease (Keith, 1959). At the present time some 160 case reports have been published, and in many of these apical left ventricular 'aneurysms' have been reported (Lang, Phillips, and McAfee, 1958; Sabiston, Neill, and Taussig, 1960; Kaunitz, 1947; Fisher and Lloyd, I95I). In the majority, the aneurysm takes the form of thinning and akinesis of the apical portion of the left ventricle, while in the case reported by Sabiston et al. (1960) paradoxical expansion was observed at the time of operation. This report describes a patient with anomalous origin of the left coronary artery from the pulmonary artery in whom the aneurysm was unusual in that it appeared as a localized diverticulum of the left ventricle which was observed to contract during ventricular systole.

\section{Case report}

The patient was first seen at the age of 3 years when, after an attack of bronchitis, a chest $x$-ray revealed cardiac enlargement. She was asymptomatic. Abnormal findings were confined to the cardiovascular system. The cardiac impulse was

t hyperdynamic, the apex being in the anterior axillary line. Auscultation revealed a single second heart sound and a grade $2 / 6$ ejection systolic

\footnotetext{
${ }_{1}^{1}$ Present address: 2nd Propedentic Medical Unit, University of Athens, Evangelismos Hospital, Ipsilanton St., Athens, Greece.
}

murmur maximal at the base. Electrocardiogram: sinus rhythm, $\mathrm{T}$ wave inversion in leads $\mathrm{I}$ and $\mathrm{aVL}$, and $Q$ waves with $S T$ elevation and $T$ wave in. version in V4-V6 (Fig. IA). Cardiac catheterization showed slight increase in pulmonary artery pressure $(32 / 15 \mathrm{mmHg})$ and indirect left atrial (wedge) pressure $(a=10, x=8, v=17, y=7$, mean $=$ I I $\mathrm{mmHg}$ ). No intracardiac shunt was detected. Venous angiography showed generalized enlargement of the left ventricle with a triangular apical aneurysm (Fig. 2). A presumptive diagnosis of anomalous origin of the left coronary artery from the pulmonary artery was made.

The patient was reinvestigated at the age of 13 years. She remained symptom free with a normal effort tolerance. Chest $x$-ray no longer showed cardiac enlargement. Electrocardiogram: $Q$ waves with $T$ wave inversion in leads $I$ and aVL with normal $Q R S$ voltage in $V_{I}-V_{3}$ but low amplitude sintered QRS complexes in V4-V6 (Fig. IB). Cardiac catheterization showed raised pulmonary artery $(45 / 20 \mathrm{mmHg})$ and left ventricular enddiastolic $(20 \mathrm{mmHg}$ ) pressures. There was also a rise in right ventricular end-diastolic pressure ( 18 $\mathrm{mmHg}$ ) with a commensurate rise in right atrial pressure $(a=20, x=13, v=19, y=13 \mathrm{mmHg}$ ). Cineaortography showed a large and tortuous right coronary artery which was seen to drain to the pulmonary artery via anastomoses with an anomalously arising left coronary artery. A pulmonary arteriogram did not opacify the left coronary artery. An injection of contrast medium into the left ventricle indicated generalized enlargement of the left ventricle with reduced emptying. There was mild mitral insufficiency. There was an aneurysm of the apex of the left ventricle (Fig. 3A) which, during ventricular systole, was seen to contract, forming a tonguelike 'diverticulum' of the left ventricle (Fig. 3B). 
FIG. I Electrocardiogräm $(A)$ at age of 3 years, $(B)$ at age of 13 years.

\section{Discussion}

Probably some 85 per cent of patients with anomalous origin of the left coronary artery die during infancy (Keith, 1959), and in such patients findings include generalized enlargement of the left ventricle with patchy fibrosis, particularly involving the apex which may be pathologically thin. There is also some degree of endocardial fibroelastosis. In a small proportion of patients who have a good collateral circulation between the left and right coronary arteries survival into adult life is possible. In such patients there is generalized enlargement of the left ventricle, and mitral incompetence may occur which may be due to dilatation of the mitral valve ring (Burchell and Brown, 1962) or to involvement of the papillary muscles by myocardial fibrosis. As was first postulated by Brooks (1885), flow in the left coronary artery is retrograde and such retrograde flow was first shown angiographically by Lampe and Verheugt in 1960. The patient presented here had all these features and, in addition, an apical left ventricular aneurysm. Thinning of the apical myocardium (Lang et al., 1958) or actual aneurysm formation with paradoxical expansion (Sabiston et $a l .$, 1960) has been reported. The patient reported here had an apical aneurysm but this was shown to contract during ventricular systole. Congenital diverticula of the left ventricle occur and may exhibit contraction during ventricular systole (Skapinker, 1951; Gasul, Arcilla, and Lev, I966); however, such diverticula are usually associated with defects in the pericardium, diaphragm, or abdominal wall (Potts, DeBoer, and Johnson, 1953); in the case described by Swyer, Mauss, and Rosenblatt (1950) there were no such defects, but the diverticulum arose from the posterior aspect of the ventricle, and in this case may truly have represented an out-pouching in an area of muscle weakness. In other cases of diverticulum of the left ventricle the defect is present from birth. Thus, the development of the aneurysm over a period of years in the case reported here, its location at the apex of the left ventricle - the usual site of thinning and aneurysm formation in patients with anomal-

FIG. 2 Left ventricle opacified by venous angiocardiogram at age of 3 years showing large (and poorly functioning) left ventricular cavity with early aneurysm formation at apex.
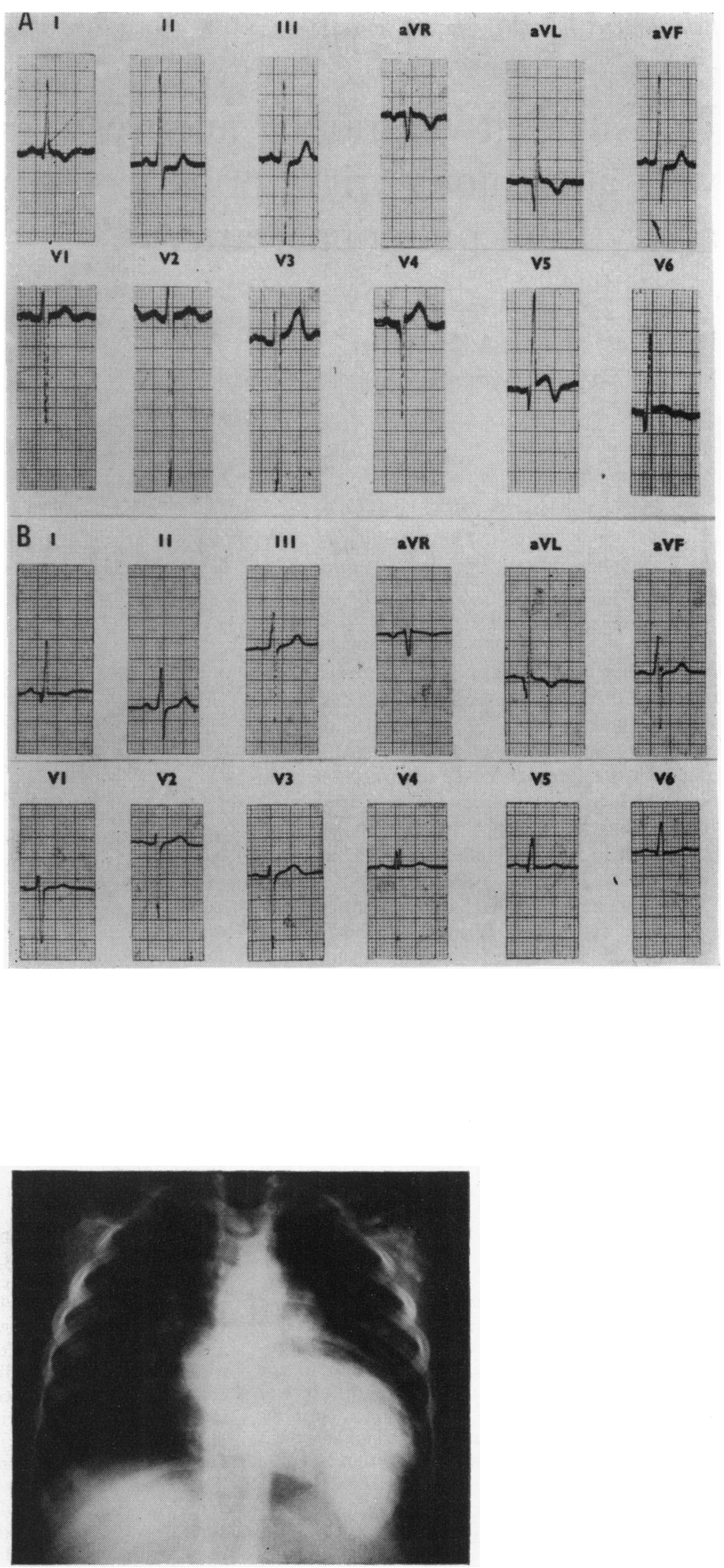


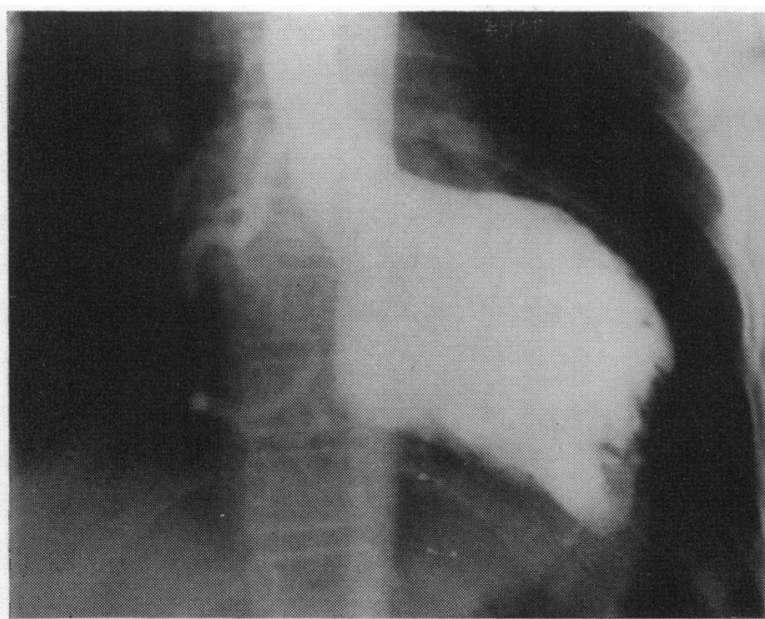

A

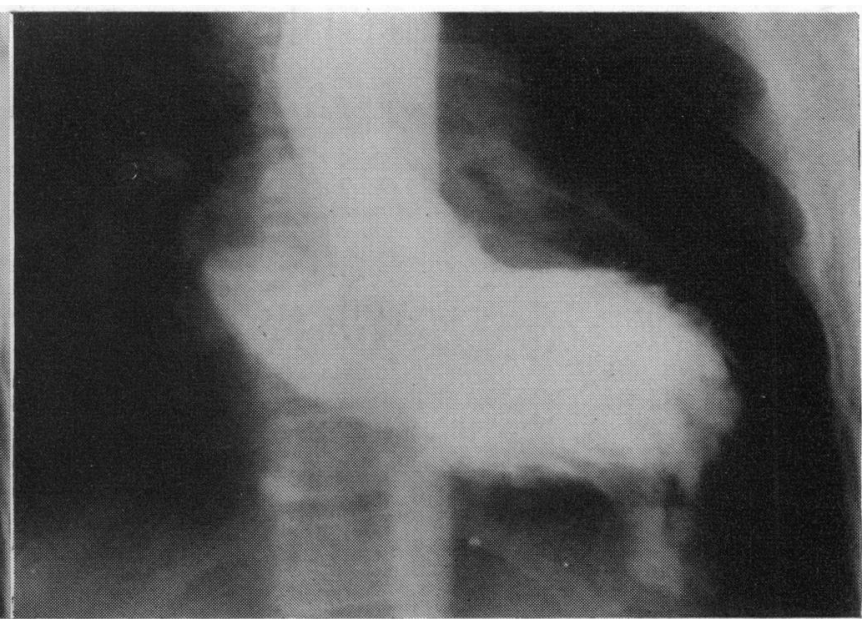

$\mathrm{B}$

FIG. 3 Left ventricular angiocardiogram at age of 13 years. $(A)$ Diastolic phase showing large left ventricular cavity and single large (right) coronary artery; the apical aneurysm is not obvious during diastole. (B) Systolic phase showing 'diverticulum-like' aneurysm obvious during ventricular systole; there is some opacification of the left atrium indicating the presence of mitral incompetence.

ous left coronary - and the absence of defects in the abdominal wall suggest that the aneurysm developed at the site of myocardial fibrosis. Such obvious aneurysm formation has not been described before and its apparent contraction during systole was a surprising feature.

We are indebted to Dr. R. V. Gibson for permission to publish details of this case.

\section{References}

Brooks, H. St J. (1885). Two cases of an abnormal coronary artery of the heart arising from the pulmonary artery; with some remarks upon the effect of this anomaly in producing cirsoid dilatation of the vessels. Fournal of Anatomy and Physiology, 20, 26.

Burchell, H. B., and Brown, A. L. (1962). Anomalous origin of coronary artery from pulmonary artery masquerading as mitral insufficiency. American Heart fournal, 63, 388.

Fisher, H., and Lloyd, O. C. (1951). A case of anomalous left coronary artery. British Heart fournal, 13, 406.

Gasul, B. M., Arcilla, R. A., and Lev, M. (1966).
Heart Disease in Children, p. 1025. Lippincott, Philadelphia.

Kaunitz, P. E. (1947). Origin of left coronary artery from pulmonary artery. Review of the literature and report of two cases. American Heart fournal, 33, 182.

Keith, J. D. (1959). The anomalous origin of the left coronary artery from the pulmonary artery. British Heart fournal, $21,149$.

Lampe, C. F. J., and Verheugt, A. P. M. (1960). Case reports. Anomalous left coronary artery. Adult type. American Heart fournal, 59, 769.

Lang, E. K., Phillips, L. A., and McAfee, J. G. (1958). Angiocardiographic features of the Bland-WhiteGarland syndrome. American fournal of Roentgenology, 80, 381.

Potts, W. J., DeBoer, A., and Johnson, F. R. (1953). Congenital diverticulum of the left ventricle. Case report. Surgery, 33, 301.

Sabiston, D. C., Neill, C. A., and Taussig, H. B. (1960). The direction of blood flow in anomalous left coronary artery arising from the pulmonary artery. Circulation, 22, 591.

Skapinker, S. (195I). Diverticulum of the left ventricle of the heart. Review of the literature and report of a successful removal of the diverticulum. Archives of Surgery, 63, 629.

Swyer, A. J., Mauss, I. H., and Rosenblatt, P. (1950). Congenital diverticulosis of left ventricle. American fournal of Diseases of Children, 79, I I I. 